Case Report

\title{
Hepatic Artery Mycotic Aneurysm Associated with Staphylococcal Endocarditis with Successful Treatment: Case Report with Review of the Literature
}

\author{
Dhara Chaudhari, ${ }^{1}$ Atif Saleem, ${ }^{2}$ Pranav Patel, ${ }^{1}$ Sara Khan, ${ }^{1}$ \\ Mark Young, ${ }^{2}$ and Gene LeSage ${ }^{2}$ \\ ${ }^{1}$ Department of Internal Medicine, East Tennessee State University, Quillen College of Medicine, 1008 Quality Circle, Apartment 79, \\ Johnson City, TN 37615, USA \\ ${ }^{2}$ Department of Gastroenterology, East Tennessee State University, Quillen College of Medicine, 1008 Quality Circle, Apartment 79, \\ Johnson City, TN 37615, USA
}

Correspondence should be addressed to Dhara Chaudhari; chaudhari@etsu.edu

Received 19 March 2013; Accepted 17 April 2013

Academic Editors: H. Komatsu, H. Miura, and N. Snyder

Copyright (C) 2013 Dhara Chaudhari et al. This is an open access article distributed under the Creative Commons Attribution License, which permits unrestricted use, distribution, and reproduction in any medium, provided the original work is properly cited.

Mycotic hepatic artery aneurysm is a vascular pathology associated with bacterial endocarditis. It is rare in occurrence after the introduction of effective antibiotics. We present a young patient with injection drug abuse associated staphylococcal endocarditis which was successfully treated with antibiotics and valve replacement who presented with abdominal pain. He was found to have mycotic aneurysm of hepatic artery which was successfully treated with coil embolization.

\section{Introduction}

Hepatic artery aneurysm is an uncommon vascular lesion. Mycotic hepatic artery aneurysm is rare but recognized complication of bacterial endocarditis. The incidence of mycotic aneurysm has decreased following the widespread use of effective antibiotics. We present a case of hepatic mycotic aneurysm in a patient with staphylococcal bacterial endocarditis.

\section{Case}

A 27-year-old male presented with complain of chest pain and abdominal pain. Patient had recent history of Methicillin Resistant Staphylococcus aureus (MRSA) aortic valve endocarditis and septic emboli to coronary arteries requiring double vessel bypass grafting with aortic valve replacement 5 months ago. He also had history of hepatitis C. He denied dyspnea, orthopnea, paroxysmal nocturnal dyspnea, fever, or chills. He also denied melena, hematochezia, or change in bowel habits. He had previous history of smoking and self-injecting drug abuse. Family history and review of system were unremarkable. Physical exam was significant for systolic heart murmur $3 / 6$ in intensity, otherwise unremarkable systemic examination. His home medicines included aspirin, carvedilol, plavix, lisinopril, and sertraline. Laboratory data revealed white blood cell count (WBC) $10.5 / \mathrm{L}$, potassium $(\mathrm{K}) 3.1 \mathrm{meq} / \mathrm{l}$, total bilirubin $0.5 \mathrm{mg} / \mathrm{dL}$, aspartate transaminase/alanine transaminase $12 / 10 \mathrm{IU} / \mathrm{L}$, and alkaline phosphate level $75 \mathrm{U} / \mathrm{L}$; urine drug screen positive for cannabinoids; chest $\mathrm{X}$ ray: no acute lung process; electrocardiogram (EKG): no acute ST-T changes. The patient was admitted to rule out acute coronary event, and infectious disease was consulted for further treatment recommendation. Computed tomography (CT) abdomen/pelvis was ordered for unexplained abdominal pain which revealed possible hepatic pseudo-aneurysm and CT Abdomen/pelvis angiography revealed right hepatic artery aneurysm of $1.2 \times 1.0 \mathrm{~cm}$ size with no flow distal to aneurysm and left hepatic artery aneurysm of $5 \times 6 \mathrm{~mm}$ size (Figure 1). Patient underwent 


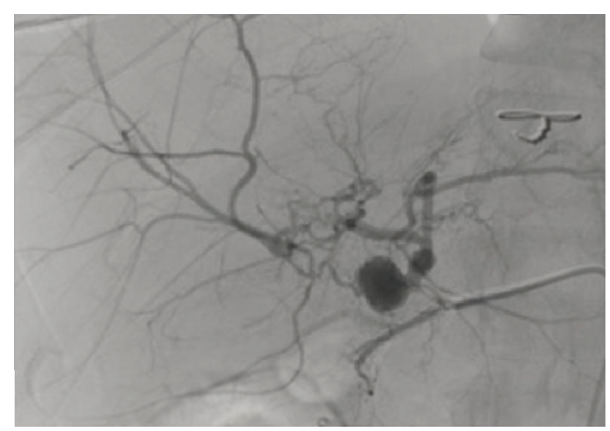

FIGURE 1: Hepatic angiography showing right hepatic artery aneurysm $(1.2 \times 1.0 \mathrm{~cm})$ and left hepatic artery aneurysm $(5 \times 6 \mathrm{~mm})$.

coiling and successful embolization with nine coils in right hepatic artery aneurysm and four coils in left hepatic artery aneurysm (Figure 2).

\section{Discussion}

Mycotic aneurysm is defined as an infectious break in the wall of an artery with formation of a blind, secular outpouching, that is, contiguous with the arterial lumen. The common sites of involvement in descending order are aorta, peripheral artery, cerebral artery, and visceral arteries [1]. Among the visceral arteries, superior mesenteric artery and arteries in liver, spleen, kidneys, or lungs has been reported in the literature [2]. Mycotic hepatic artery aneurysm is a rare but important vascular pathology accounting for $0.1 \%$ of all arterial aneurysms and $20 \%$ of all visceral aneurysms [3]. Mycotic aneurysm was first described by Osler in 1885. The term "mycotic" is a misnomer as fungal infections are uncommonly associated with this pathology but was used as it looks like fungal growth due to beaded appearance and multiple aneurysms [4].

In preantibiotic era, most of hepatic artery aneurysms (HAA) were mycotic and associated with bacterial endocarditis [5]. Most common causative pathogens are Streptococcus and Staphylococcus groups. There are few cases reported in the literature of mycotic hepatic artery aneurysm with staphylococcal endocarditis [5-9]. Also methicillin resistant strain of staphylococcus is more pronounced in intravenous drug users. Our patient had staphylococcus endocarditis secondary to injection drug abuse which we believe was the primary risk factor leading to formation of the mycotic aneurysm. In addition, Gram-negative bacteria, fungal infection with Aspergillus or Candida, and Mycobacteria are rare causes of mycotic aneurysm [1].

After introduction of antibiotics, incidence of mycotic aneurysm has decreased [5]. Currently, most of the hepatic artery aneurysms are primary, likely due to medial degeneration or secondary atherosclerosis. Less commonly, polyarteritis nodosa, trauma, fibromuscular dysplasia, and infectious pathology like acute pancreatitis or cholecystitis have been reported in the literature [10]. Immunocompromised states such as immunodeficiency, diabetes, or malignancy can also predispose to mycotic aneurysm [1]. Although rare,

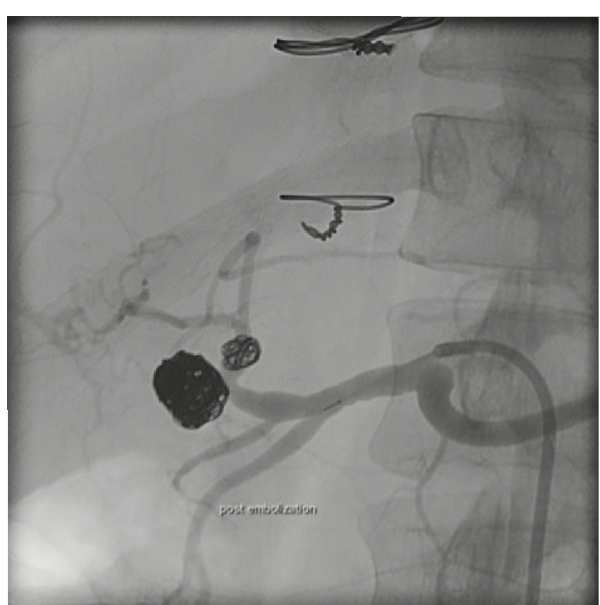

FIGURE 2: Postembolisation celiac angiography showing successful coiling of both aneurysms.

hepatic artery aneurysms have also been reported after liver transplantation [11]. Stengal and Wolferth in 1923 reported 217 patients with mycotic aneurysm, of which 187 had endocarditis and 19 patients had hepatic artery aneurysm [12]. This contrasts with a more recent study from the Mayo clinic, which documented 306 patients with true visceral aneurysm diagnosed between 1980 and 1999 with 36 patients (12\%) with hepatic artery aneurysm and only one patient with hepatic mycotic aneurysm [13].

The pathogenesis of mycotic aneurysm can be explained by lodging of infected emboli into lumen of normal vessel leading to inflammation and necrosis, followed by weakening of the arterial wall causing dilation [14]. Other elucidated etiologies could be circulating organisms targeting preexisting vascular defect or contiguous vessel involved from adjacent infectious source $[1,14]$. Hepatic artery aneurysm may involve intrahepatic (25\%), extrahepatic (75\%), or common hepatic artery. The common hepatic artery is the most frequent site $(63 \%)$, followed by the right hepatic artery $(28 \%)$, the left hepatic artery (5\%), and both hepatic arteries (4\%) [15]. In our case, aneurysms were in both right and left hepatic arteries.

Although most hepatic artery aneurysms are asymptomatic [13], when symptomatic $80 \%$ of patients present with right upper quadrant pain. Gastrointestinal hemorrhage [16] and jaundice [9] can be associated with the abdominal pain. The triad of epigastric pain, hemobilia, and obstructive jaundice suggestive of Quincke's symptom are seen in onethird of patients $[17,18]$.

Hepatic artery aneurysm has high risk of rupture especially mycotic aneurysm because of dislodgment of infected emboli causing vessel wall inflammation and necrosis. It may rupture directly into the intestinal tract presenting as either upper or lower gastrointestinal bleeding or can rupture into peritoneal cavity presenting with symptoms of peritonitis $[16,17]$. When it ruptures into adjacent portal or hepatic venous system, the patient can present with jaundice.

The widespread use of ultrasonography especially color doppler ultrasonography or CT scan has resulted in increased 
identification of asymptomatic hepatic artery aneurysms [8, 10]. A multidetector CT scan can provide three-dimensional anatomy of the visceral arteries [19]. MR angiography is an alternative for patients who cannot have CT angiography. The gold standard test is conventional angiography which provides accurate location, size, and shape of the aneurysm. There have been sporadic cases reported of PET scan detecting arterial aneurysms [1].

Treatment of hepatic artery mycotic aneurysm depends on size, location/anatomy, and comorbidity. Treatment is recommended in symptomatic patients who have a higher risk of rupture and aneurysm with a diameter greater than $2 \mathrm{~cm}$. Treatment modalities are embolization with coils or endovascular stenting and surgery with or without revascularisation [20]. Embolization with coils is the treatment of choice for intrahepatic lesion, high-risk surgical candidates, secular aneurysm, and proximal aneurysm. Surgical options include ligation, ligation excision, and hepatic lobe resection. Surgical options are suitable to patients with low risk and usually distal aneurysm [10].

\section{Conclusion}

Mycotic hepatic aneurysm is a rare entity in antibiotic era. Because of their asymptomatic nature, high index of suspicion is required for diagnosis. The patients with recent or remote history of endocarditis or sepsis are at risk. Mycotic aneurysm, if not treated, is associated with high-risk rupture and increased mortality.

\section{Conflict of Interests}

The authors declare no conflict of interests.

\section{References}

[1] W. K. Lee, P. J. Mossop, A. F. Little et al., "Infected (mycotic) aneurysms: spectrum of imaging appearances and management," Radiographics, vol. 28, no. 7, pp. 1853-1868, 2008.

[2] L. M. Messina and C. J. Shanley, "Visceral artery aneurysms," Surgical Clinics of North America, vol. 77, no. 2, pp. 425-442, 1997.

[3] G. A. Kim, H. C. Lee, Y. J. Jin et al., "A case of ruptured mycotic hepatic artery aneurysm successfully treated using arterial embolization," Yeungnam University Journal of Medicine, vol. 29, no. 1, pp. 24-27, 2012.

[4] B. H. Stack, J. T. Rankin, and R. J. Bentley, "Hepatic artery aneurysm after staphylococcal endocarditis," British Medical Journal, vol. 3, no. 5619, pp. 659-660, 1968.

[5] M. Jordan, S. Razvi, and M. Worthington, "Mycotic hepatic artery aneurysm complicating Staphylococcus aureus endocarditis: successful diagnosis and treatment," Clinical Infectious Diseases, vol. 39, no. 5, pp. 756-757, 2004.

[6] K. Mojab, L. T. Lim, F. Esfahani, and L. Portela, "Mycotic aneurysm of the hepatic artery causing obstructive jaundice," American Journal of Roentgenology, vol. 128, no. 1, pp. 143-144, 1977.

[7] L. L. Porter, M. C. Houston, and S. Kadir, "Mycotic aneurysms of the hepatic artery: treatment with arterial embolization," The American Journal of Medicine, vol. 67, no. 4, pp. 697-701, 1979.
[8] A. N. Sukerkar, C. C. Dulay, E. Anandappa, and S. Asokan, "Mycotic aneurysm of the hepatic artery. Case diagnosed with radionuclide imaging and ultrasound," Radiology, vol. 124, no. 2, p. 444, 1977.

[9] A. B. Tierney and F. J. Palmer, "Mycotic aneurysm of the right hepatic artery presenting with obstructive jaundice," Australasian Radiology, vol. 31, no. 1, pp. 50-52, 1987.

[10] P. Hulsberg, J. L. Garza-Jordan, R. Jordan, P. Matusz, R. S. Tubbs, and M. Loukas, "Hepatic aneurysm: a review," The American Surgeon, vol. 77, no. 5, pp. 586-591, 2011.

[11] V. S. Jones, M. S. Chennapragada, D. J. E. Lord, M. Stormon, and A. Shun, "Post-liver transplant mycotic aneurysm of the hepatic artery," Journal of Pediatric Surgery, vol. 43, no. 3, pp. 555-558, 2008 .

[12] A. Stengal and C. C. Wolferth, "Mycotic aneurysms of intravascular origin," Archives of Internal Medicine, vol. 31, no. 4, pp. 527-554, 1923.

[13] M. A. Abbas, R. J. Fowl, W. M. Stone et al., "Hepatic artery aneurysm: factors that predict complications," Journal of Vascular Surgery, vol. 38, no. 1, pp. 41-45, 2003.

[14] Y. Nakata, S. Shionoya, and K. Kamiya, "Pathogenesis of mycotic aneurysm," Angiology, vol. 19, no. 10, pp. 593-601, 1968.

[15] I. W. Park, T. W. Park, S. G. Han et al., "Delayed rupture of mycotic hepatic artery aneurysm in a patient with infective mitral endocarditis-first case in Korea," Korean Circulation Journal, vol. 37, no. 1, pp. 43-46, 2007.

[16] R. Dogan, E. Yildirim, and S. Gokturk, "Gastrointestinal hemorrhage caused by rupture of a pseudoaneurysm of the hepatic artery," The Turkish Journal of Gastroenterology, vol. 23, no. 2, pp. 160-164, 2012.

[17] N. N. Harlaftis and J. T. Akin, "Hemobilia from ruptured hepatic artery aneurysm: report of a case and review of the literature," American Journal of Surgery, vol. 133, no. 2, pp. 229-232, 1977.

[18] J. T. Stauffer, M. D. Weinman, and T. E. Bynum, "Hemobilia in a patient with multiple hepatic artery aneurysms: a case report and review of the literature," American Journal of Gastroenterology, vol. 84, no. 1, pp. 59-62, 1989.

[19] C. C. Kibbler, D. L. Cohen, J. K. Cruicshank et al., "Use of CAT scanning in the diagnosis and management of hepatic artery aneurysm," Gut, vol. 26, no. 7, pp. 752-756, 1985.

[20] A. B. Christie, D. B. Christie III, D. K. Nakayama, and M. M. Solis, "Hepatic artery aneurysms: evolution from open to endovascular repair techniques," The American Surgeon, vol. 77, no. 5, pp. 608-611, 2011. 


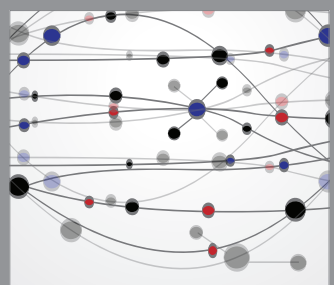

The Scientific World Journal
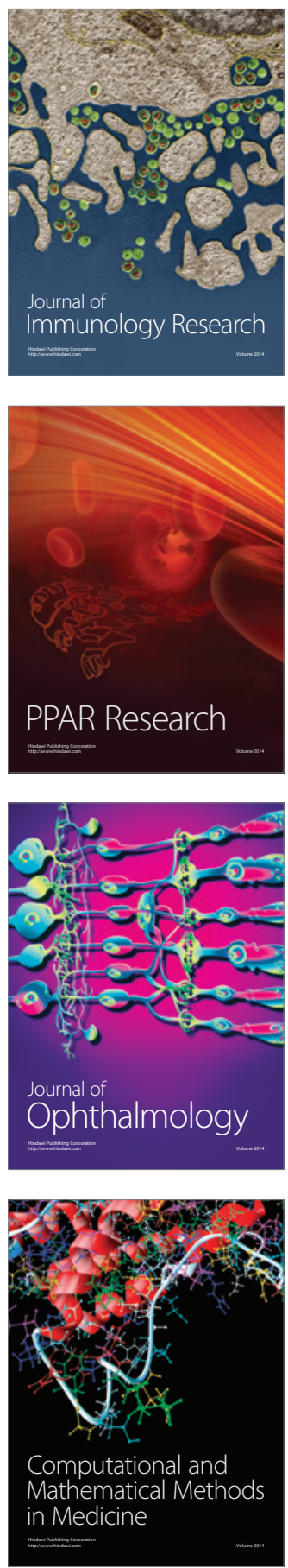

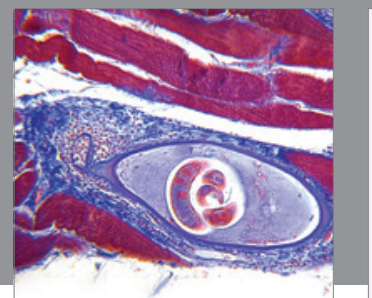

Gastroenterology

Research and Practice
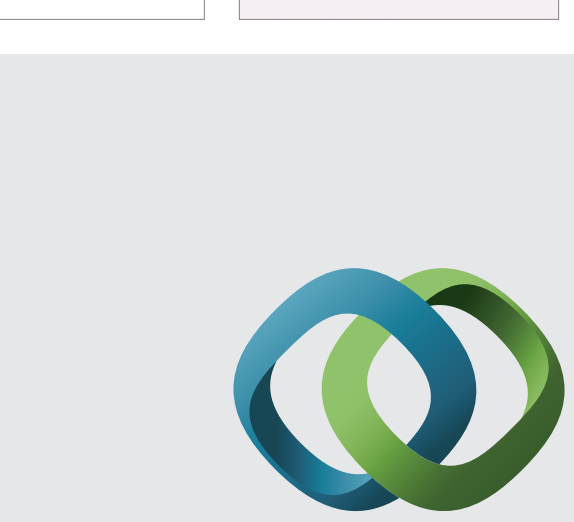

\section{Hindawi}

Submit your manuscripts at

http://www.hindawi.com
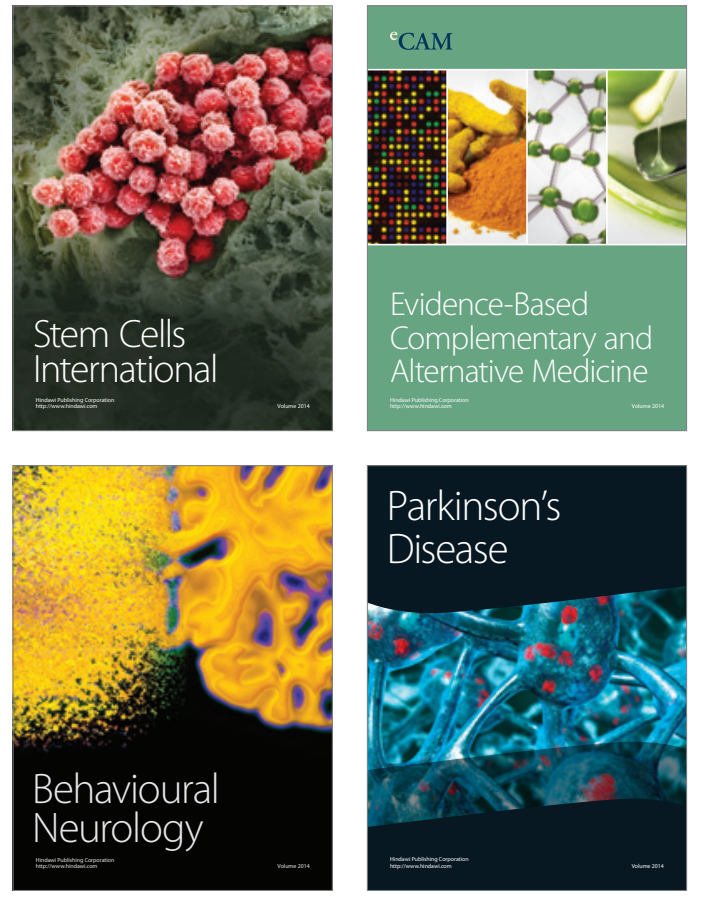
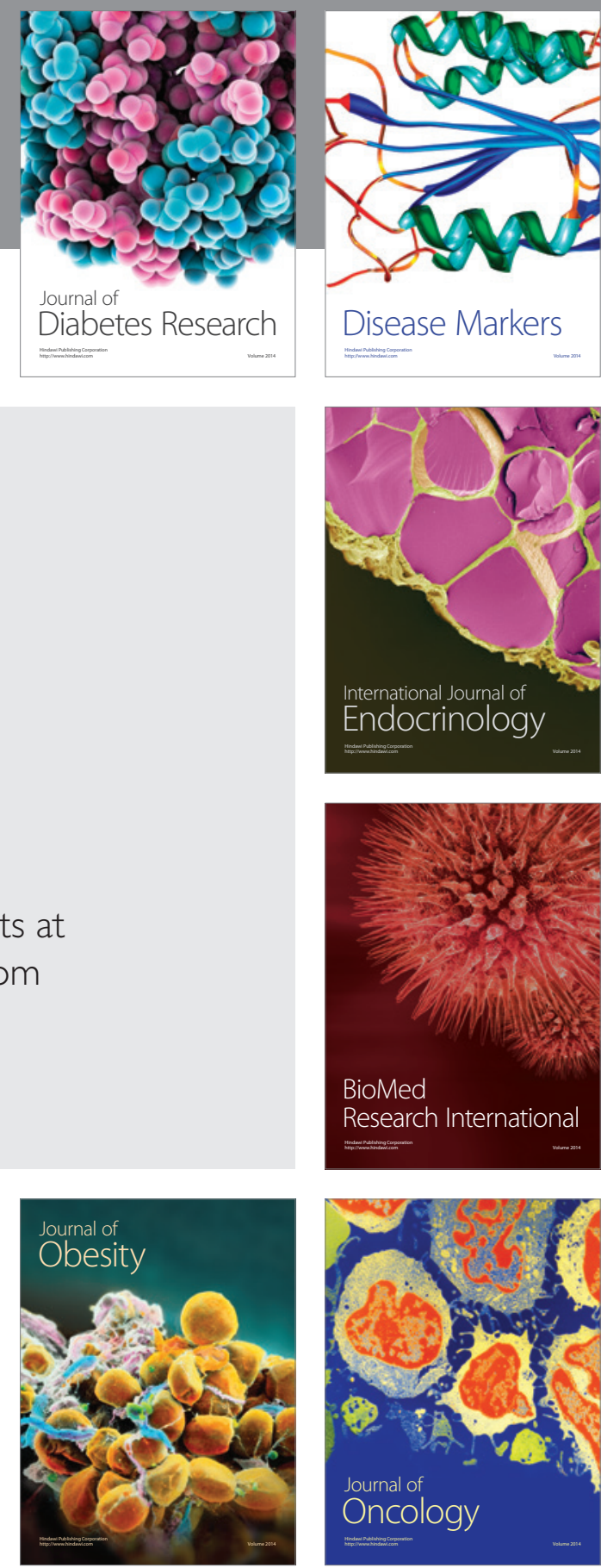

Disease Markers
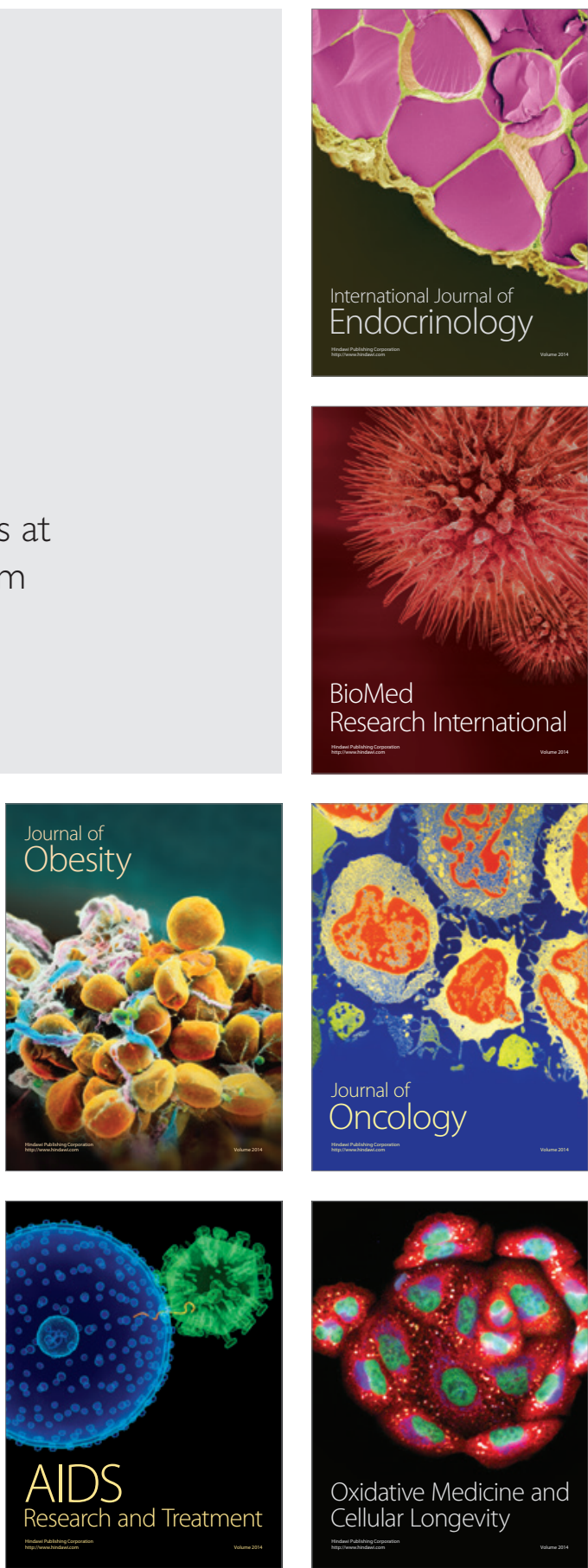